\title{
Simultaneous Navigation and SAR Auto-focusing
}

\author{
Zoran Sjanic \\ Department of Electrical Engineering \\ Division of Automatic Control \\ Linköping University \\ Linköping, Sweden \\ zoran@isy.liu.se
}

\author{
Fredrik Gustafsson \\ Department of Electrical Engineering \\ Division of Automatic Control \\ Linköping University \\ Linköping, Sweden \\ fredrik@isy.liu.se
}



Figure 1: Illustration of the back-projection method for creating of the SAR images. Figure is not to scale.

process is illustrated in Figure 1 where two points are imaged. Each column of the low resolution real aperture radar image on the bottom is back-projected to the sub images, which means that column is mapped to the two-dimensional sub image. These sub images are in turn summed to the final synthetic aperture radar image on the top. The simulation plots of this setup is also depicted in Figure 2. Even in this process it is assumed that the radar antenna's flown path is linear with constant altitude and heading, but the method can be extended to non-linear tracks as well. However exact inversion is not guaranteed. The main disadvantage of this method is the large amount of operations required to create an image which is proportional to $\mathcal{O}\left(N \times M^{2}\right)$ for $M \times M$ pixels image using an aperture with $N$ positions. However by means of coordinate transformation, an approximated solution to exact back-projection can be performed which is called Fast Factorised Back- 


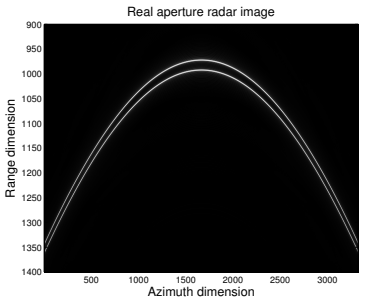

(a) Real aperture radar image of the two points.

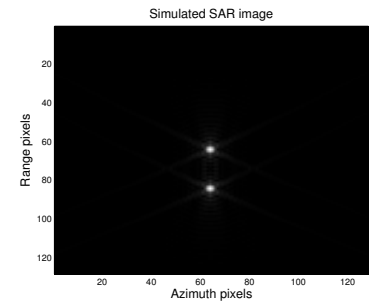

(b) Synthetic aperture radar image of the two points.

Figure 2: Real and synthetic aperture radar images of the two point scene.

projection, see [7]. This algorithm is proportional to $M^{2} \log N$ operations, which for large $N$ implies an important saving. With this faster algorithm it should be possible to create images in real time, possibly in dedicated hardware. Since back-projection algorithms are dependent on knowledge of antenna's position in order to get focused images, the image focus can be measured and used for estimation of the trajectory. An

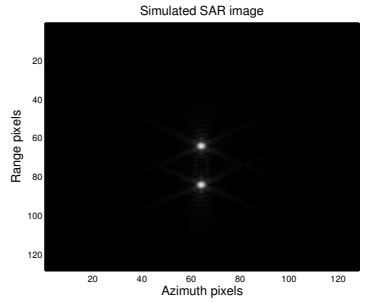

(a) Focused SAR image of two point targets.

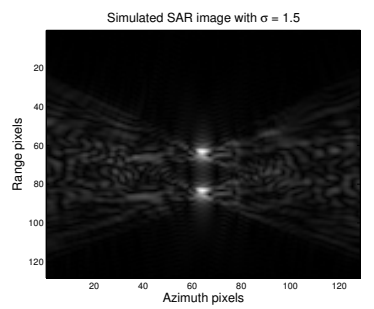

(c) Unfocused SAR image of (d) Unfocused SAR image of two point targets with $\sigma=1.5$. two point targets with $\sigma=3$.

Figure 3: Example SAR images with different perturbed trajectories.

example of this is depicted in Figure 3, where two point targets are imaged. In Figure 3a, a linear path is simulated, which results in a perfectly focused image. In the other three images the variation in range position was added as $\mathcal{N}\left(0, \sigma^{2}\right)$ where $\sigma=\{0.5,1.5,3\}$ and the images are created with an assumption that the path was linear. This gives unfocused images as depicted. Traditional methods for auto-focusing are mostly open-loop type methods where either SAR images or raw radar data are used, for example Phase Gradient Auto-focus (PGA), [6, 8, 3]. The significant common denominator for all these methods is that the image is created with assumptions on linear flight trajectory and focusing is done afterwards in an open-loop way discarding eventual flight path information. This is a consequence of the off-line image generating process where the trajectory is no longer interesting. In the setup where SAR
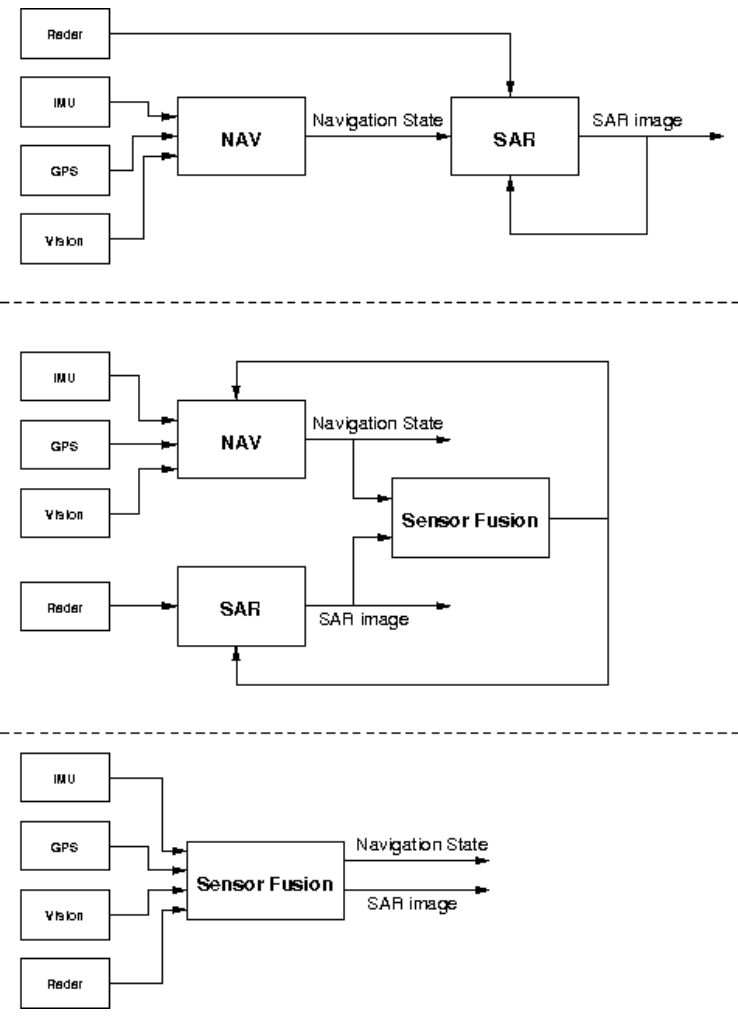

Figure 4: Top: SAR architecture where navigation data is used in an open-loop manner. Middle: SAR architecture where navigation and SAR data is used together in a decentralised sensor fusion framework. Bottom: SAR architecture where navigation and SAR data is used together in a centralised sensor fusion framework.

images are generated on-line an idea is to use information from the image unfocusing and navigation system together and in a sensor fusion framework try to obtain the best solution to both focusing and navigation simultaneously. This can be implemented in a centralised or decentralised manner, as depicted in Figure 4.

\section{Sensor Fusion Framework}

In order to use a sensor fusion framework, the usual approach is to define a discrete time dynamical model of the system

$$
\begin{aligned}
x_{t+1} & =f\left(x_{t}, u_{t}, w_{t}\right) \\
y_{t} & =h\left(x_{t}, u_{t}\right)+e_{t}
\end{aligned}
$$


where $x_{t}$ are states of the system, $u_{t}$ are known inputs, $w_{t}$ is system noise with variance $Q_{t}, e_{t}$ is measurement noise with variance $R_{t}, y_{t}$ are measurements, $f$ describes dynamics of the system and $h$ is the measurement equation that relates measurements and states of the system. In this case, the dynamics of the system can be usual 6 -DOF aircraft dynamics. Since focus of the image depends on the unknown trajectory, one solution is to solve following minimisation problem

$$
\begin{aligned}
& \left.\min _{x_{0}, w_{1: N}} \gamma_{F} F\left(x_{0: N}\right)+\gamma_{s} \sum_{k=1}^{N} \| y_{k}-h\left(x_{k}, u_{k}\right)\right) \|_{R_{k}}^{2} \\
& \text { s.t. } x_{t+1}=f\left(x_{t}, u_{t}, w_{t}\right)
\end{aligned}
$$

where $\gamma_{F}$ and $\gamma_{s}$ are the weights. In Equation (2a), $F\left(x_{0: N}\right)$ is a function of the SAR image $I$ created from the radar measurements and is of the type "how focused is the image?". Since the SAR image is a function of the trajectory $x_{0: t}, F$ is also function of it. For the purpose of determining $F$ some measure of the image focus must be used. This is the subject of Section 4 .

\section{System model}

In this setup following 2-DOF INS time discrete dynamics is used, [2], as a specialisation of the general dynamics (1a):

$$
\begin{aligned}
& x_{t+1}=F x_{t}+G w_{t} \\
& F=\left[\begin{array}{ccc}
I_{2} & T_{s} I_{2} & \frac{T_{s}^{2}}{2} I_{2} \\
0_{2 \times 2} & I_{2} & T_{s} I_{2} \\
0_{2 \times 2} & 0_{2 \times 2} & I_{2}
\end{array}\right] \\
& G=\left[\begin{array}{c}
\frac{T_{s}^{3}}{6} I_{2} \\
\frac{T_{s}^{2}}{2} I_{2} \\
T_{s} I_{2}
\end{array}\right] \\
& x_{t}=\left[\begin{array}{llllll}
X_{t} & Y_{t} & v_{t}^{X} & v_{t}^{Y} & a_{t}^{X} & a_{t}^{Y}
\end{array}\right]^{T} \\
& w_{t}=\left[\begin{array}{ll}
w_{t}^{X} & w_{t}^{Y}
\end{array}\right]^{T}
\end{aligned}
$$

where $T_{s}$ is the sampling time, $X$ is the position in azimuth direction and $Y$ is the position in range direction, $v^{X}$ and $v^{Y}$ are the velocities in the $X$ - and $Y$-directions respectively and $a^{X}$ and $a^{Y}$ are the accelerations in $X$ and $Y$-directions respectively. This model is used for the whole trajectory. Since this model is linear and time invariant the stationary Kalman filter can be used to estimate $x_{t}$ giving $\hat{x}_{t}$ and its corresponding covariance $P_{t}$. The covariance of the estimate, due to the fact that system is time invariant and linear, will converge to the stationary covariance $\bar{P}$ which can be calculated as

$\bar{P}=F \bar{P} F^{T}-F \bar{P} H^{T}\left(H \bar{P} H^{T}+R\right)^{-1} H \bar{P} F^{T}+G Q G^{T}$

where $F$ and $G$ are defined above, and $H$ is the linearised measurement equation $h\left(x_{t}, u_{t}\right)$ (in our simulation case it is chosen as $H=I_{6}$ since we assume that all

\begin{tabular}{|c||c|c|}
\hline Parameter & Accuracy $(1-\sigma)$ & Stat. acc. $(1-\sigma)$ \\
\hline \hline Position & $3 \mathrm{~m}$ & $0.093 \mathrm{~m}$ \\
\hline Velocity & $0.4 \mathrm{~m} / \mathrm{s}$ & $0.012 \mathrm{~m} / \mathrm{s}$ \\
\hline Acceleration & $0.06 \mathrm{~m} / \mathrm{s}^{2}$ & $0.015 \mathrm{~m} / \mathrm{s}^{2}$ \\
\hline
\end{tabular}

Table 1: Accuracy and stationary accuracy for the navigation parameters

states are measured). For a typical navigation system used in an UAV, the performance for these parameters (assumed to be measurement noise) can be summarised according to Table 1 and $Q$, which represents disturbance on states like wind turbulence, can be taken as $\operatorname{diag}\{0.25,0.25\}$. With these values, the stationary covariance is as given in the third column in Table 1.

\section{Focus Measures}

One common focus measure in SAR or image processing literature is image entropy calculated as

$$
F_{1}(I)=-\sum_{k=1}^{256} p_{k} \log _{2}\left(p_{k}\right)
$$

where $p_{k}$ is an approximated grey level distribution of the $M \times N$ grey-scale image $I_{i j}=\left|\tilde{I}_{i j}\right|$ where $\tilde{I}_{i j}$ is the complex-valued SAR image and it can be obtained from the image histogram calculated as

$$
\begin{aligned}
p_{k} & =\frac{\left\{\# \text { of pixel values }\left|\tilde{I}_{i j}\right|\right\} \in[k-1, k]}{M N} \\
k & \in[1,256]
\end{aligned}
$$

The more focused the image is, the lower the entropy is, see for example [4] or [9]. Histograms for the images in Figure 3 are given in Figure 5. Note the log-scale on the $y$-axis. An alternative definition of entropy is, [10],

$$
\begin{aligned}
F_{2}(\tilde{I}) & =-\sum_{i=1}^{M} \sum_{j=1}^{N} p_{i j} \ln \left(p_{i j}\right) \\
p_{i j} & =\frac{\left|\tilde{I}_{i j}\right|^{2}}{\sum_{i=1}^{M} \sum_{j=1}^{N}\left|\tilde{I}_{i j}\right|^{2}}
\end{aligned}
$$

where a complex-valued SAR image $\tilde{I}$ is used directly instead of the grey-scale SAR image $I$.

\subsection{Focus Measure Performance}

Entropy 1 and 2 focus measures are tested and compared on a SAR image according to Figure 6 and the results are depicted in Figure 7 where standard deviations $1-\sigma, 2-\sigma$ and $3-\sigma$ are also drawn. This image is chosen since it is more informative than the image in Figure 3. In this simulation setup the state noise in model (3) is set to zero, i.e. the trajectory is completely deterministic. This is done in order to illustrate the focus measure functions $F_{i}$ in a two dimensional 

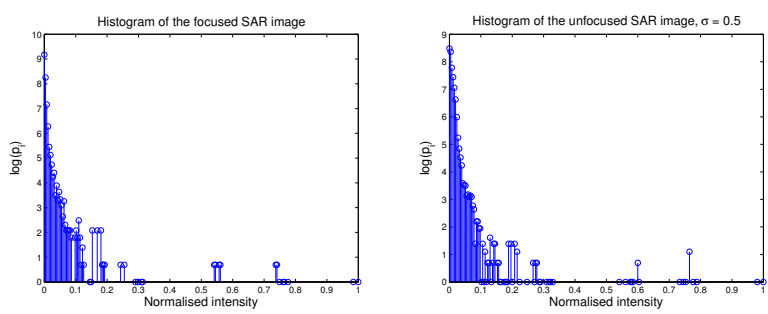

(a) Histogram of the focused image.

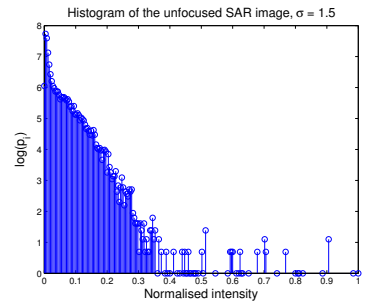

(c) Histogram of the unfocused image with $\sigma=1.5$.

Figure 5: Histograms for the images in Figure 3.

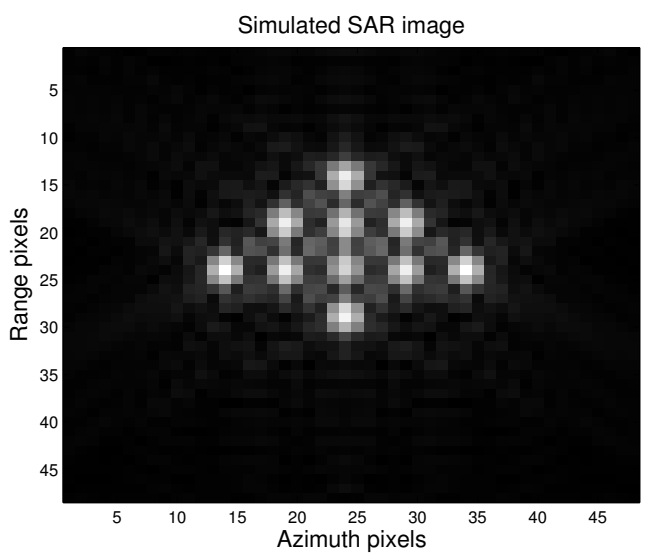

Figure 6: SAR image with a more informative scene than in Figures 2 and 3.

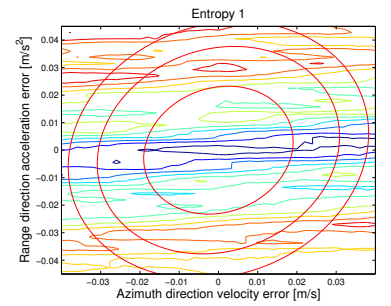

(a) Entropy 1 focus measure.

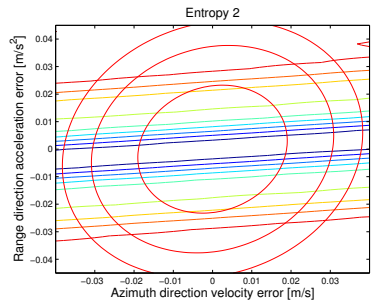

(b) Entropy 2 focus measure.
Figure 7: Focus measures for the image in Figure 6 with standard deviation ellipses.

plot, since the trajectory, and consequently the focus measure, is then only dependent on the initial values. In these figures it can be seen that entropy 2 has a convex and pretty nice behaviour around the true value of the initial state. However it looks very flat along the velocity direction which indicates that it is very difficult to estimate that particular state. The entropy 1 measure has, on the other hand, a sharp minimum for the correct value of the initial state but many local minima. This means that the two entropy measures complement each other perfectly, and can be used in combination to obtain the global minimum of the focus measure.

\section{Search Methods}

As demonstrated in Section 4.1, the entropy 2 measure can be used to, with a local minimisation method, for example gradient search, come close to the global minimum and then entropy 1 can be used to obtain the global minimum. Note also that only the focus measure is used to find estimate of the states i.e. $\gamma_{s}$ is set to zero while $\gamma_{F}$ is set to one in Equation (2a).

\subsection{Gradient Search}

As mentioned above, the measure $F_{2}$ can be used as a first step to get near the global minimum, since it appears to be convex. However it is very flat in the proximity of global minimum, and then some other measure can be used to find the global minimum, for example $F_{1}$ due to its sharp minimum for correct values. Since focus of the images strongly depends only on the two initial states, $v_{0}^{X}$ and $a_{0}^{Y}$, a gradient search can be in that case formulated as Gauss-Newton search

$$
\begin{aligned}
x^{i+1} & =x^{i}-\mu_{i} R\left(x^{i}\right)^{-1} \nabla F\left(x^{i}\right) \\
x^{0} & =\left[\begin{array}{c}
\hat{v}_{0}^{X} \\
\hat{a}_{0}^{Y}
\end{array}\right] \\
\nabla F(x) & =\left[\begin{array}{c}
\frac{\partial F}{\partial v_{0}^{X}} \\
\frac{\partial F}{\partial a_{0}^{Y}}
\end{array}\right] \\
R(x) & =\nabla F(x) \cdot(\nabla F(x))^{T}
\end{aligned}
$$

and $\mu_{i}$ is a step size with $\mu_{0}=1$. An initial estimate, $x^{0}$, can be taken from the navigation system. In some 
cases, $R$ can be singular or close to singular and this would cause problems. In this case the search procedure is modified to Levenberg-Marquardt procedure where $R$ is calculated as

$$
R(x)=\nabla F(x) \cdot(\nabla F(x))^{T}+\lambda I
$$

and $\lambda$ is chosen as a small number, usually $\lambda \sim 10^{-6}$.

In order to demonstrate the behaviour of the gradient search for this setup, a simulation of the search procedure is illustrated in Figures 9 and 10. Here, the algorithm is initiated with different starting points $x^{0}=\left[\begin{array}{ll}v_{0}^{X}, & a_{0}^{Y}\end{array}\right]^{T}$ based on the stationary covariance of the states in the system. Those values

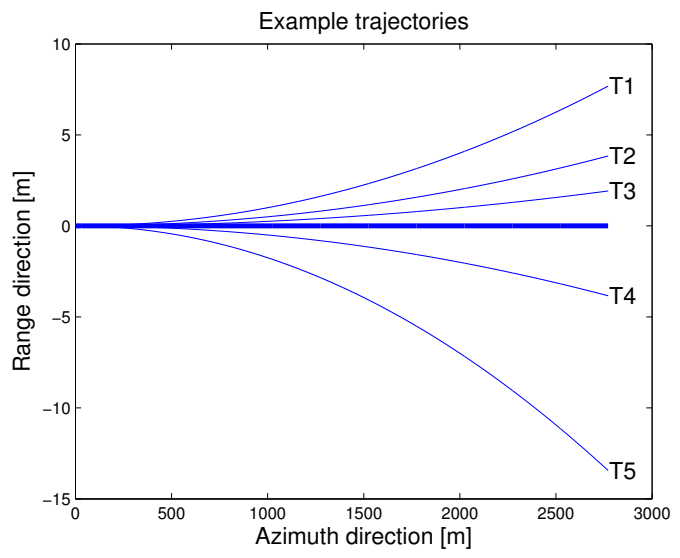

Figure 8: Trajectory examples for different initial values of the velocity and acceleration.

are $[100.005,0.005]^{T},[99.99,0.01]^{T},[99.995,0.02]^{T}$, $[100.02,-0.01]^{T}$ and $[100.005,-0.035]^{T}$. The trajectories generated with these initial values are illustrated in Figure 8. In Figure 9, the gradient search based on the entropy measure $F_{2}$ is illustrated and we can see that the solutions converge to the flat ridge-like area close to the correct acceleration, but not necessarily to the correct velocity. In Figure 10, a gradient search where entropy measure $F_{1}$ is used is depicted. In this case, the algorithm is initiated with the solution from the entropy $F_{2}$ search. It can be seen that this minimisation strategy works pretty well, although one solution is stuck in a local minimum. In that case the velocity error is the largest one of all errors. It is interesting to see how the image created with the solution that is stuck in the local minima of the entropy measure $F_{1}$ looks like compared to the unfocused image that it started with. As illustrated in Figure 11, it can be seen that the image created with values from the minimisation procedure is very close to the focused image and much better than the unfocused image that it started with. The probable explanation for this comes from the fact that small azimuth direction velocity errors do not influence the final image much due to the quantisation effects. However the estimate of the navigation state is not correct.

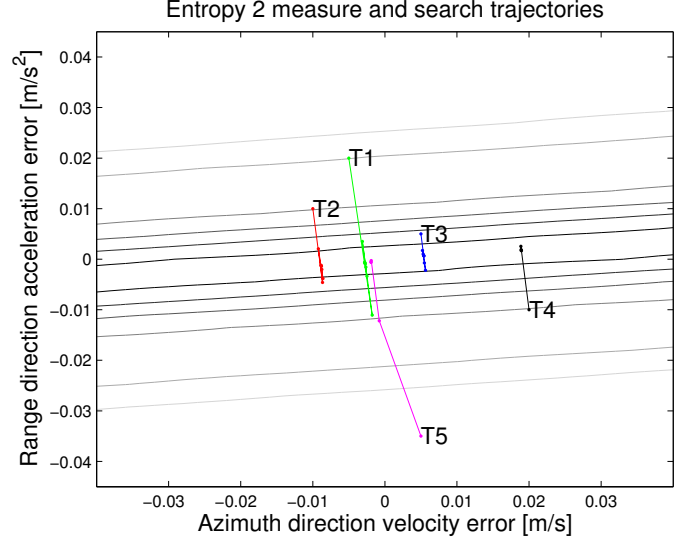

Figure 9: Search trajectory for five different values of $x^{0}$ on entropy 2 focus measure.

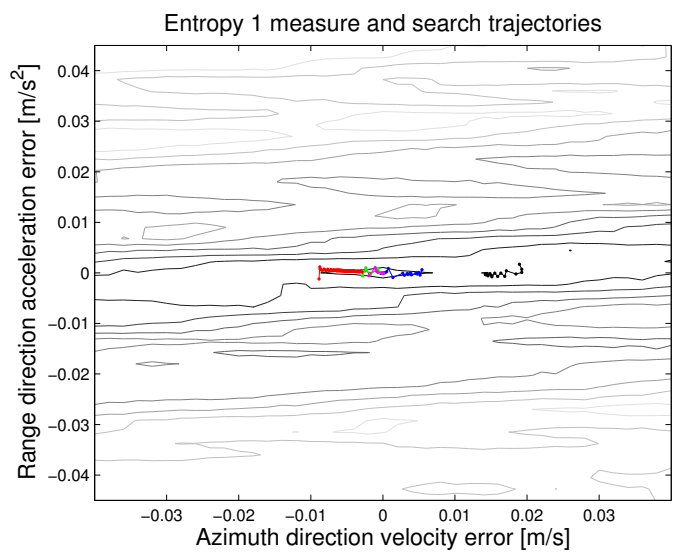

Figure 10: Search trajectory for entropy 1 focus measure with $x^{0}$ given by the entropy 2 gradient search.

\subsection{Global Grid Search}

As seen in Section 5.1, local search methods will not work completely satisfactory on this problem and some kind of global method must be used if global minimum is sought. The most straightforward approach is to use grid search and find global minimum of the loss function (2a). This can be done in an iterative manner where the last iteration's result is an input to the next. The iterations are initialised with the state estimates from the navigation system or pure measurements if all states are measured, and the initial grid size can be chosen based on the state estimate covariance or measurement noise covariance. The grid size can be changed between iterations since it is assumed that each iteration makes the estimate better. The performance of such an estimator will depend on the grid resolution which in turn directly influences how much time the estimation takes, so there is a fundamental balance between performance and speed. A trajectory with initial values $\left[\begin{array}{lllllll}0 & 0 & 99.99 & 0 & 0 & 0.01\end{array}\right]^{T}$ is simulated and radar measurements are collected along it. These 

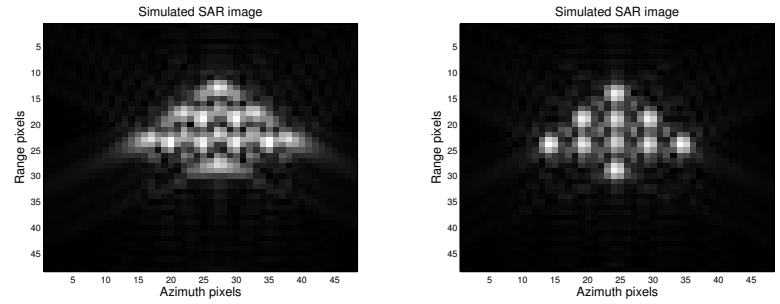

(a) Image created with error in (b) Image created with error in velocity of $0.02 \mathrm{~m} / \mathrm{s}$ and in ac- velocity of $0.014 \mathrm{~m} / \mathrm{s}$ and in acceleration of $-0.01 \mathrm{~m} / \mathrm{s}^{2}$. celeration of $-0.0003 \mathrm{~m} / \mathrm{s}^{2}$.

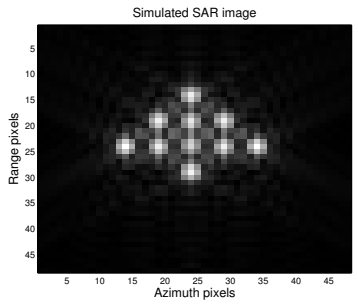

(c) Focused image as a reference.

Figure 11: Resulting images from the minimisation procedure with starting point $[100.02,-0.01]^{T}$.

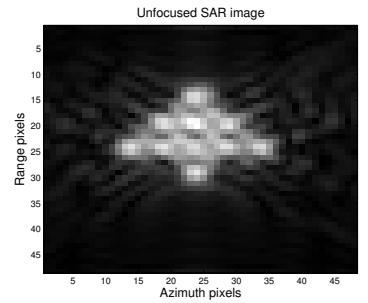

(a) Unfocused SAR image generated with noisy trajectory.

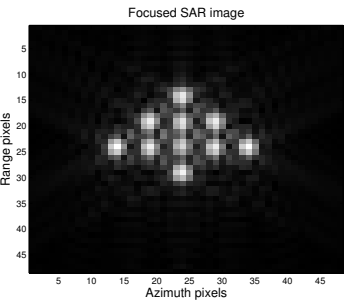

(b) Focused SAR image generated with estimated trajectory with two free parameters.



(c) Focused SAR image generated with estimated trajectory with four free parameters.

Figure 12: Resulting images generated from the minimisation procedure based on a grid method. values are chosen just to obtain a trajectory with some movement. Measurement noise is added to the trajectory and an image is created with this noisy trajectory. The obtained image is according to Figure 12a. The trajectory is then estimated with the procedure above where only initial states are estimated and the trajectory is then calculated with the dynamical model (3). The state noise $w_{t}$ is set to zero in this setup. In Figure $12 \mathrm{~b}$ only $v_{0}^{X}$ and $a_{0}^{Y}$ are set as free parameters and the others are set to zero, i.e. the true values. In Figure 12c both $X$ - and $Y$-direction initial velocities and accelerations are free parameters while initial positions are set to zero. The estimate of the initial states is $\left[\begin{array}{ll}\hat{v}_{0}^{X} & \hat{a}_{0}^{Y}\end{array}\right]^{T}=\left[\begin{array}{lll}99.996 & 0.011\end{array}\right]^{T}$ in the first case and $\left[\begin{array}{llll}\hat{v}_{0}^{X} & \hat{v}_{0}^{Y} & \hat{a}_{0}^{X} & \hat{a}_{0}^{Y}\end{array}\right]^{T}=\left[\begin{array}{llll}99.891 & 0.015 & 0.005 & 0.008\end{array}\right]^{T}$. It can be seen that both image and state estimate are worse if four parameters are set free, which is natural since the problem is much harder in that case. The quality of the state estimate and image focusing is also dependent on the grid resolution which in this simulation case was not so fine due to the execution time.

\section{Conclusions and Future Work}

Here we presented the method which is based on the decentralised sensor fusion framework and demonstrated that it has potential of obtaining more focused images and fairly accurate estimates of the navigation states. As it can be seen from the simulation examples of the simple scene and the usage of the entropy focus measure, the method for simultaneous auto-focusing and estimation of the navigation states works fairly well although not perfect. It looks like there are some fundamental limitations in the possibility to estimate all states accurately from the focus measure, as in the case of velocity in azimuth direction. Nevertheless, even in the case where the velocity estimate is not so good, the image is much more focused than the one which is started with, so the method works fairly well if only focused images are the goal.

The next step to take is to use more of the centralised sensor fusion framework and use raw radar measurement data directly similar to the well known Phase Gradient Auto-focus. This approach leads to more filteringlike methods, where EKF or similar can be used to obtain the estimates of the states.

\section{References}

[1] L. J. Cutrona, W. E. Vivian, E. N. Leith, and G. O. Hall. A high-resolution radar combat-surveillance system. Military Electronics, IRE Transactions on, MIL-5(2):127-131, April 1961.

[2] J. Farrell and M. Barth. The global positioning system and inertial navigation. McGraw-Hill Professional, 1999. 
[3] Mengdao Xing and Xiuwei Jiang and Renbiao Wu and Feng Zhou and Zheng Bao. Motion Compensation for UAV SAR Based on Raw Radar Data. Geoscience and Remote Sensing, IEEE Transactions on, 47(8):2870-2883, Aug. 2009.

[4] R.L.Jr. Morrison and D.C.Jr. Munson. An experimental study of a new entropy-based sar autofocus technique. In Image Processing. 2002. Proceedings. 2002 International Conference on, volume 2, pages II-441-II-444 vol.2, 2002.

[5] F. Natterer. The Mathematics of Computerised Tomography. New York: Wiley, 1986.

[6] Chris Oliver and Shaun Quegan. Understanding Synthetic Aperture Radar Images. The SciTech Radar and Defense Series. SciTech, 2004.

[7] L.M.H. Ulander, H. Hellsten, and G. Stenstrom. Synthetic-aperture radar processing using fast factorized back-projection. Aerospace and Electronic Systems, IEEE Transactions on, 39(3):760-776, July 2003.

[8] Wahl, D.E. and Eichel, P.H. and Ghiglia, D.C. and Jakowatz, C.V., Jr. Phase gradient autofocus-a robust tool for high resolution SAR phase correction. Aerospace and Electronic Systems, IEEE Transactions on, 30(3):827-835, Jul 1994.

[9] Li Xi, Liu Guosui, and Jinlin Ni. Autofocusing of ISAR images based on entropy minimization. Aerospace and Electronic Systems, IEEE Transactions on, 35(4):1240-1252, Oct 1999.

[10] A.F. Yegulalp. Minimum entropy SAR autofocus, 1998. 